Discrete Comput Geom 34:87-96 (2005)

DOI: $10.1007 / \mathrm{s} 00454-004-1151-1$

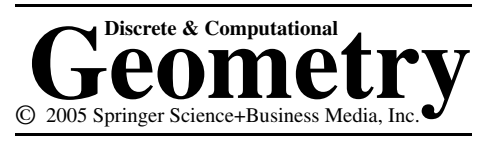

\title{
Equipartitions of Measures by 2-Fans
}

\author{
Sergey Bereg \\ Department of Computer Science, University of Texas at Dallas, \\ Box 830688, Richardson, TX 75083, USA \\ besp@utdallas.edu
}

\begin{abstract}
We study the problem of computing an equitable 2-fan for three masses distributed on the 2-sphere. The existence of an equitable 2-fan was shown by Bárány and Matoušek [3]. The contribution of this paper is twofold. (i) We prove the existence of an infinite set of equitable 2-fans. (ii) We present an efficient algorithm for finding an equitable 2-fan when the mass distributions are discrete, i.e. finite sets of points. Both (i) and (ii) can be easily extended to mass distributions in the plane instead of the sphere.
\end{abstract}

\section{Introduction}

Balanced partitions of sets of points and disssections of mass distributions are a fundamental topic in Combinatorial Geometry [23] and received attention recently [3], [4], [6], [7], [22]. An equipartition of a mass distribution (measure) $\mu$ defined on a space $X$ is a finite collection $\mathcal{K}=\left\{K_{1}, \ldots, K_{q}\right\}$ of measurable sets forming a partition (dissection) of $X$ such that $\mu\left(K_{i}\right)=\mu\left(\mathbb{R}^{d}\right) / q$ for each $i=1, \ldots, q$. We assume that the space $X$ is either the plane $\mathbb{R}^{2}$ or the unit 2-sphere. We are interested in the partitions by $k$-fans [3], see Fig. 1 for examples. Equipartitions find applications in computer science [19] (for example, geometric range searching), in statistics [5] (for example, regression depth) and in "practice" [1] (for example, cutting a cake).

3-Fan. Kaneko and Kano [15] conjectured that, for any $q n$ red and $q m$ blue points ( $q, n, m>0$ are integers) in the plane in general position, there are $q$ disjoint convex polygons with $n$ red and $m$ blue points in each of them. The conjecture of Kaneko and Kano has been independently proven by Bespamyatnikh et al. [6], Ito et al. [14] (the case $q=3$ ) and Sakai [20]. If $q=3$, the partition is a 3-fan with convex wedges.

Bárány and Matoušek [3] established a number of results concerning the existence (and non-existence) of balanced $k$-fans. In particular, they proved the existence of a 2 -fan equipartitioning three masses on the sphere and a 3 -fan equipartitioning two masses on the sphere. 


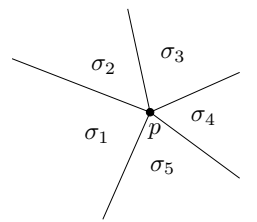

(a)

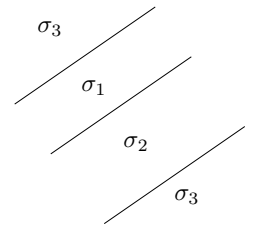

(b)

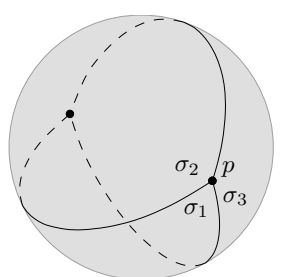

(c)

Fig. 1. (a) A 5-fan in the plane with the center $p$, (b) a 3-fan in the plane and (c) a 2-fan on the sphere with the center $p$.

Equipartitioning by a $k$-fan is related to the Ham Sandwich Theorem [2], [23]. The Ham Sandwich problem is well studied from the algorithmic point of view [10], [12], [13], [17], [21], [24]. An optimal algorithm of Lo et al. [17] finds a Ham Sandwich cut in linear time. Very recently, Bose et al.[7] studied the problem of finding a geodesic ham-sandwich cut which can be viewed as a generalization of the classical one.

We consider the problem of finding an equitable 2-fan for three discrete masses defined as finite sets of points. The brute-force approach based on the existence of a 2-fan [3] is to check all posssible 2-fans. There are $O\left(n^{5}\right)$ combinatorially different 2-fans for a set of $n$ points. It would be useful for designing an efficient algorithm to prove the existence of a an equatable 2-fan that satisfies some property. Mikio Kano conjectured [16] that every line contains the center of an equitable 2-fan. In this paper we prove the conjecture.

Theorem 1. Given a set of points in the plane colored in red/blue/green such that (i) the points are in general position, and (ii) the number of points of each color is even, there exists an equitable 2-fan with center on a given line.

We prove Theorem 1 in a slightly stronger form. We assume that points on a sphere are in general position if (i) no three points lie on the same great circle, and (ii) no two points are antipodal (i.e. their midpoint is the sphere center).

Theorem 2. Given a set of points on the unit 2-sphere colored in red/blue/green such that (i) the points are in general position, and (ii) the number of points of each color is even, there exists an equitable 2-fan with center on a given great circle.

This result can be stated in a more general setting: For any three finite Borel measures on the 2-sphere $S$, any Jordan curve on $S$ whose ends are antipodal points contains the center of an equitable 2 -fan.

The proof of Theorem 2 allows us to reduce the problem of finding an equitable 2-fan to the well-known problem of slope selection [9]. Slope selection can be solved optimally in $O(n \log n)$ time using a deterministic algorithm [8], [9] and a randomized algorithm (and somewhat simpler) [11], [18]. We present an algorithm for finding an equitable 2-fan on the unit 2-sphere. The algorithm runs in $O\left(N \log ^{2} N\right)$ time where $2 N$ is the total number of points. 


\section{Preliminaries}

Let $S=\left\{p \mid p_{x}^{2}+p_{y}^{2}+p_{z}^{2}=1\right\}$ be the unit sphere in $\mathbb{R}^{3}$. Let $p$ be a point of $S$. For a direction $d$ perpendicular to $O p$, we denote by $c(p, d)$ the great half-circle between $p$ and $-p$ in the direction $d$. Let $d_{1}$ and $d_{2}$ be two distinct directions perpendicular to $O p$. The two great $\operatorname{arcs} c\left(p, d_{1}\right)$ and $c\left(p, d_{2}\right)$ are considered to be a 2 -fan $f=\left(p ; d_{1}, d_{2}\right)$. They partition the sphere into two regions-lunes of the sphere-denoted as $\sigma_{1}(f)$ and $\sigma_{2}(f)$, see Fig. 1(c).

Continuous Masses. Suppose that $\lambda_{1}, \lambda_{2}, \lambda_{3}$ are absolutely continuous Borel probabilities on $S$ such that all the measures are positive on non-void open sets (as in [4]). For a point $p$ and a direction $d_{1}$, there is a unique 2-fan $f\left(p ; d_{1}, d_{2}\right)$ such that $\lambda_{1}\left(\sigma_{1}(f)\right)=\frac{1}{2}$ (also there is a unique 2-fan such that $\left.\lambda_{1}\left(\sigma_{1}(f)\right)+\lambda_{2}\left(\sigma_{1}(f)\right)+\lambda_{3}\left(\sigma_{1}(f)\right)=\frac{3}{2}\right)$. Let $X$ be the set of all such 2-fans. We can associate a 2-frame (or just frame) with a 2-fan. Thus, the space of 2-fans is homeomorphic to Stiefel manifold $V_{2}\left(\mathbb{R}^{3}\right) \cong S O(3)$ of all orthonormal 2-frames in $\mathbb{R}^{3}$.

Discrete Masses. From now on, we assume that the masses are discrete and colored: let $R, B$ and $G$ be sets of red, blue and green points on the sphere $S$, respectively. We assume that the set of each color contains an even number of points, namely $|R|=2 n,|B|=2 m$ and $|G|=2 k$. Let $P=R \cup B \cup G$ be the set of all given points and let $N=n+m+k$.

Let $\mathcal{A}$ be the sphere arrangement generated by the great circles passing through pairs of points of $P$. Let $p$ be a point from the interior of a face in $\mathcal{A}$ and let $d$ be a direction such that $c(p, d)$ avoids $P$. We define a canonical 2-fan $f=\left(p ; d, d^{\prime}\right)$ for the pair $(p, d)$ so that the number of points in $P \cap \sigma_{1}(f)$ is $N$ (note that the direction $d^{\prime}$ is not unique but the set $P \cap \sigma_{1}(f)$ is unique). For simplicity, we call the canonical 2-fan just the 2 -fan, denote it by $\varphi(p, d)$, and denote its lunes by $\sigma_{1}(p, d)$ and $\sigma_{2}(p, d)$. We call $p$ a center of the 2 -fan and $d$ a starting direction of the 2 -fan.

Two 2-fans $\varphi=(p, d)$ and $\varphi^{\prime}=\left(p, d^{\prime}\right)$ are called neighbors if the interior of one of the two lunes between $c(p, d)$ and $c\left(p, d^{\prime}\right)$ contains exactly one data point. Let $\sigma\left(p, d, d^{\prime}\right)$ denote the lune between $c(p, d)$ and $c\left(p, d^{\prime}\right)$ obtained by rotating $c(p, d)$ about the line passing through the origin $O$ and $p$ in clockwise order when we look from $p$ towards $O$. A 2-fan $\varphi^{\prime}=\left(p, d^{\prime}\right)$ is called a $C W$-neighbor of $\varphi=(p, d)$ if exactly one data point lies in the interior of $\sigma\left(p, d, d^{\prime}\right)$. A 2 -fan $\varphi^{\prime}=\left(p, d^{\prime}\right)$ is called a $C C W$-neighbor of $\varphi=(p, d)$ if exactly one data point lies in the interior of $\sigma\left(p, d^{\prime}, d\right)$.

Let

$$
\delta(x)= \begin{cases}0, & x=0 \\ x /|x|, & x \neq 0\end{cases}
$$

\section{The Existence of an Equitable 2-Fan}

To evaluate the quality of a 2 -fan $(p, d) \in X$ we define a map $\mu: X \rightarrow \mathbb{Z}^{2}$ by $\mu(p, d)=$ $\left(\mu_{1}(p, d), \mu_{2}(p, d)\right)$ where $\mu_{1}(p, d)$ is the number of red points in $\sigma_{1}(p, d)$ and $\mu_{2}(p, d)$ is the number of blue points in $\sigma_{1}(p, d)$. For detecting if a 2 -fan is equitable we introduce a 


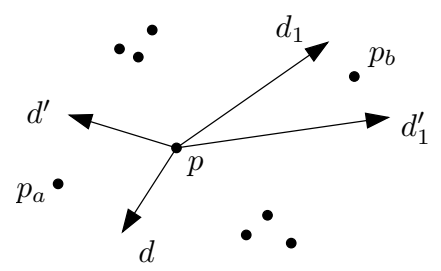

Fig. 2. The 2-fans $\varphi=\left(p ; d, d_{1}\right)$ and $\varphi^{\prime}=\left(p ; d^{\prime}, d_{1}^{\prime}\right)$.

$\operatorname{map} \alpha: X \rightarrow \mathbb{Z}^{2}$ by $\alpha(p, d)=\left(\alpha_{1}(p, d), \alpha_{2}(p, d)\right)$ where $\alpha_{1}(p, d)=\delta\left(\mu_{1}(p, d)-n\right)$ and $\alpha_{2}(p, d)=\delta\left(\mu_{2}(p, d)-m\right)$. Clearly, a 2-fan $(p, d)$ is equitable if and only if $\alpha(p, d)=(0,0)$.

Lemma 3. Let $p$ be a point in the interior of a face $f \in \mathcal{A}$ and let $\varphi=(p, d)$ and $\varphi^{\prime}=\left(p, d^{\prime}\right)$ be two neighbor frames. If $\alpha(\varphi) \neq 0$ and $\alpha\left(\varphi^{\prime}\right) \neq 0$, then $\alpha(\varphi)$ and $\alpha\left(\varphi^{\prime}\right)$ are either equal or adjacent vertices in the label diagram depicted in Fig. 3(a).

Proof. Without loss of generality we assume that $\varphi^{\prime}$ is the CW-neighbor of $\varphi$. Let $\mu(p, d)=\left(n_{1}, n_{2}\right)$ and $\mu\left(p, d^{\prime}\right)=\left(n_{1}^{\prime}, n_{2}^{\prime}\right)$. Let $\Sigma=\sigma_{1}(p, d) \cap P$ and $\Sigma^{\prime}=\sigma_{1}\left(p, d^{\prime}\right) \cap$ $P$. The sets $\Sigma$ and $\Sigma^{\prime}$ differs by two points, say $p_{a}$ and $p_{b}$, such that $\Sigma \cup\left\{p_{b}\right\}=\Sigma^{\prime} \cup\left\{p_{a}\right\}$, see Fig. 2.

If $p_{a}$ and $p_{b}$ have the same color, then $\mu(p, d)=\mu\left(p, d^{\prime}\right)$ and $\alpha(\varphi)=\alpha\left(\varphi^{\prime}\right)$. We assume that $p_{a}$ and $p_{b}$ have different colors. Then $\left|n_{i}-n_{i}^{\prime}\right| \leq 1$ for $i=1,2$. There are eight possible values for $\alpha(p, d)$ and $\alpha\left(p, d^{\prime}\right)$-the vertices of the diagram in Fig. 3(a). It can be easily verified that the edges of the label diagram represent all possible transitions from $\alpha(p, d)$ to $\alpha\left(p, d^{\prime}\right)$.

We associate a circular diagram with the labels $\alpha()$ by placing them at the vertices of the regular 8-gon on the unit circle as shown in Fig. 3(b). Let $p$ be a point in the interior of a face $f \in \mathcal{A}$. Let $\pi(p)$ be the closed path of $\alpha(p, d)$ on the circular diagram, when

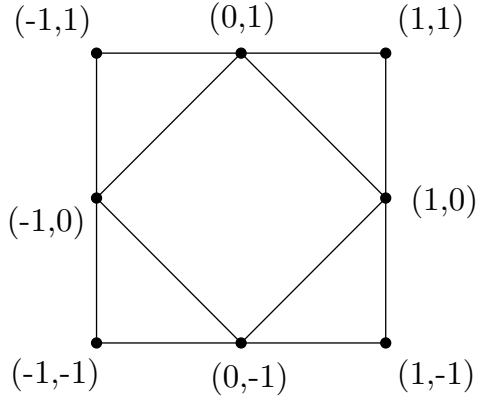

(a)

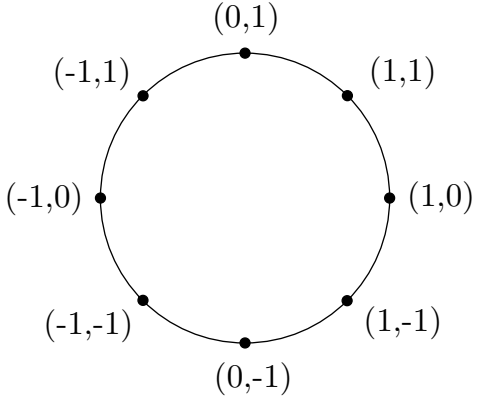

(b)

Fig. 3. Label diagram for 2 -fans. (a) Transitions. (b) Circular diagram. 
$d$ rotates $360^{\circ}$ in clockwise order. We define the winding number $\omega(p)$ as the number of clockwise turns of the path $\pi(p)$.

Lemma 4. Let $p$ be a point in the interior of a face $f \in \mathcal{A}$. If there is no equitable 2 -fan with center $p$, then $\omega(p)$ is well defined and is odd.

Proof. Suppose that there is no equitable 2-fan with center $p$. The winding number is well defined since every transition is represented in the circular diagram as $\pm \frac{1}{8}$ or $\pm \frac{1}{4}$ of the one clockwise turn. We show that $\omega(p)$ is odd. Let $\left(p ; d_{1}, d_{1}^{\prime}\right)$ be a 2fan. Let $\pi_{1}=\alpha\left(p, d_{1}\right), \alpha\left(p, d_{2}\right), \ldots, \alpha\left(p, d_{l}\right)$ be the path on the label diagram when the starting direction of a 2 -fan rotates from $d_{1}$ to $d_{l}=d_{1}^{\prime}$ in clockwise order. Note that $\alpha\left(p, d_{l}\right)=-\alpha\left(p, d_{1}\right)$. Thus, the path $\pi_{1}$ makes $t+\frac{1}{2}, t \in \mathbb{Z}$, clockwise turns on the label diagram. Let $\pi_{2}$ be the path on the label diagram when the starting direction of a 2-fan rotates from $d_{l}$ to $d_{1}$ in clockwise order. It has the property that $\pi_{2}=\alpha\left(p, d_{l}\right)=-\alpha\left(p, d_{1}\right),-\alpha\left(p, d_{2}\right), \ldots,-\alpha\left(p, d_{l}\right)=\alpha\left(p, d_{1}\right)$. Thus, $\pi_{2}$ makes $t+\frac{1}{2}, t \in \mathbb{Z}$, clockwise turns on the label diagram. The winding number $\omega(p)$ is $2 t+1$. The lemma follows.

Lemma 4 allows us to define the winding number of a face $f$, denoted by $\omega(f)$, assuming that an equitable 2-fan does not exist. Since the paths $\pi(p)$ are the same for all points $p$ in the face $f$, we denote it by $\pi(f)$.

Lemma 5. Let $e$ be an edge of the arrangement $\mathcal{A}$ and let $f_{1}$ and $f_{2}$ be two faces incident to $e$. If neither $f_{1}$ nor $f_{2}$ contains the center of an equitable 2-fan, then $\omega\left(f_{1}\right)$ and $\omega\left(f_{2}\right)$ are equal.

Proof. The edge $e$ is an arc on a great circle $C$ passing through two points of $P$, say $p_{a}$ and $p_{b}$. The points $p_{a}$ and $p_{b}$ partition the great circle $C$ into two great arcs $A_{1}$ and $A_{2}$ such that one, say $A_{1}$, is shorter than the other. If $e$ is a part of $A_{1}$, then the paths $\pi\left(f_{1}\right)$ and $\pi\left(f_{2}\right)$ are equal. Suppose that $e \subseteq A_{2}$.

Let $p_{i}, i=1,2$, be a point in the face $f_{i}$. Let $\varphi_{j}=\left(p_{1} ; d_{j}, d_{j}^{\prime}\right), j=1,2,3$, be three consecutive 2-fans $\left(\varphi_{j+1}\right.$ is the $\mathrm{CW}$-neighbor of $\left.\varphi_{j}\right)$ with center $p_{1}$ such that the lune $\sigma_{1}\left(p_{1}, d_{1}^{\prime}, d_{2}^{\prime}\right)$ contains $p_{a}$, see Fig. 4. Let $p_{c}$ and $p_{d}$ be two other points that change $\sigma_{1}$ of these three 2-fans. The points of $P$ in the lunes $\sigma_{1}$ transform between 2-fans of the path $\pi\left(f_{1}\right)$ as follows:

$$
\Sigma \cup\left\{p_{c}, p_{d}\right\} \rightarrow \Sigma \cup\left\{p_{a}, p_{c}\right\} \rightarrow \Sigma \cup\left\{p_{a}, p_{b}\right\},
$$

where $\Sigma$ is the set of points in $P$ that stay in $\sigma_{1}$. The corresponding transformations for the point $p_{2}$ are

$$
\Sigma \cup\left\{p_{c}, p_{d}\right\} \rightarrow \Sigma \cup\left\{p_{b}, p_{c}\right\} \rightarrow \Sigma \cup\left\{p_{a}, p_{b}\right\},
$$

see Fig. 4.

If $p_{a}$ and $p_{b}$ have the same color then the transformations for $p_{1}$ are the same as for $p_{2}$ and, thus, the paths $\pi\left(p_{1}\right)$ and $\pi\left(p_{2}\right)$ are equal. Suppose that the colors of $p_{a}$ and $p_{b}$ 


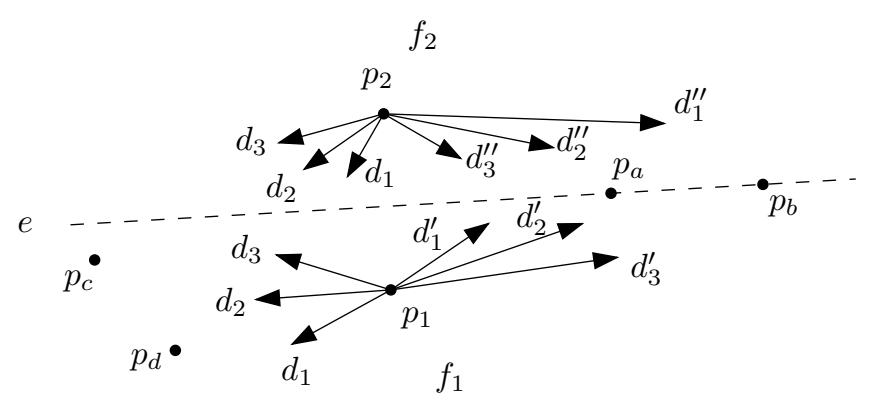

Fig. 4. Lemma 5.

are different. Without loss of generality we assume that $p_{a}$ is red and $p_{b}$ is not red. Let $n_{1}=\mu_{1}\left(p_{1}, d_{1}\right)$. It is easily verified that,

(i) if $p_{d}$ is red, then $\mu_{1}\left(p_{i}, d_{j}\right) \in\left\{n_{1}-1, n_{1}\right\}$ for all $i=1,2$ and $j=1,2,3$, and

(ii) if $p_{d}$ is not red, then $\mu_{1}\left(p_{i}, d_{j}\right) \in\left\{n_{1}, n_{1}+1\right\}$ for all $i=1,2$ and $j=1,2,3$.

Therefore, the numbers $\alpha_{1}\left(p_{i}, d_{j}\right), i=1,2, j=1,2,3$, either all avoid -1 or all avoid +1 . This implies that the paths $\alpha\left(p_{1}, d_{1}\right), \alpha\left(p_{1}, d_{2}\right), \alpha\left(p_{1}, d_{3}\right)$ and $\alpha\left(p_{2}, d_{1}\right)$, $\alpha\left(p_{2}, d_{2}\right), \alpha\left(p_{2}, d_{3}\right)$ make the same (possibly fractional) number of turns.

Note that the path $\pi\left(p_{1}\right)$ may differ from $\pi\left(p_{2}\right)$ in two places since the arc $A_{1}$ can be swept by either of the great half-circles of a 2 -fan $\left(p ; d, d^{\prime}\right)$. The case where $c\left(p, d^{\prime}\right)$ intersects $A_{1}$ is similar. The lemma follows.

The arrangement $\mathcal{A}$ is defined using $\left(\begin{array}{c}2 N \\ 2\end{array}\right)$ great circles. Clearly, for each face $f$ of $\mathcal{A}$, its symmeric image about the center of the sphere is a face of $\mathcal{A}$. We call it the opposite face of $f$.

Theorem 6 (Antipodal Mapping). Let $f_{1}$ and $f_{2}$ be two faces of the arrangement $\mathcal{A}$ such that $f_{2}$ is the opposite face of $f_{1}$. If the winding numbers $\omega\left(f_{1}\right)$ and $\omega\left(f_{2}\right)$ are defined, then $\omega\left(f_{1}\right)=-\omega\left(f_{2}\right)$.

Proof. Let $p_{1}$ be a point of the face $f_{1}$. Then $p_{2}=-p_{1}$ is the point of the face $f_{2}$. Let $q_{0}, q_{1}, \ldots, q_{2 N-1}$ be the points of $P$ in clockwise order from the point of view $p_{1}$. Let $P_{i}, 0 \leq i<2 N$, be the set $\left\{q_{i}, q_{i+1}, \ldots, q_{i+N}\right\}$ (we assume that the indices are modulo $2 N)$. Let $\pi_{i}$ denote $\left(\delta\left(\left|P_{i} \cap R\right|-n\right), \delta\left(\left|P_{i} \cap B\right|-m\right)\right)$. Then $\pi\left(p_{1}\right)=\pi_{0}, \pi_{1}, \ldots, \pi_{2 N-1}$. On the other hand $\pi\left(p_{2}\right)=\pi_{2 N-1}, \pi_{2 N-2}, \ldots, \pi_{0}$ since the clockwise order of the points of $P$ from the point of view $p_{2}$ is $q_{2 N-1}, q_{2 N-2}, \ldots, q_{0}$. Therefore the path $\pi\left(p_{2}\right)$ makes $-\omega\left(p_{1}\right)$ number of turns. The theorem follows.

Lemma 5 and Theorem 6 imply Theorems 2 . The continuous versions of Theorems 1 and 2 can be shown using standard techniques, see for example [3], [6] and [19]. 


\section{Algorithm for Finding 2-Fans}

We show that an equitable 2 -fan whose existence is guaranteed by Theorem 2 can be computed efficiently.

Theorem 7. Given a set of $2 N$ points on the 2-sphere colored in red/blue/green such that (i) the points are in general position, and (ii) the number of points of each color is even, an equitable 2-fan with center on a given great circle can be found in $O\left(N \log ^{2} N\right)$ time using $O(N)$ space.

Proof. By rotating the points on the sphere we can assume that the given great circle is $\left\{p \in S \mid p_{z}=0\right\}$. Theorem 2 implies that the great half-circle $\gamma=\{(\cos \psi, \sin \psi, 0) \mid \pi / 2$ $\leq \psi \leq 3 \pi / 2\}$ contains the center of an equitable 2-fan. Let $\gamma_{0}=(0,1,0)$ and $\gamma_{1}=$ $(0,-1,0)$ be the endpoints of $\gamma$.

Winding number. Let $p$ be a point on the sphere $S$. The winding number of $p$ can be found in $O(N \log N)$ time by (i) sorting the points of $P$ in clockwise order, and (ii) computing the path $\pi(p)$ on the label diagram (note that the algorithm can stop here if $\alpha()=0$ is found), and (iii) computing $\omega(p)$.

Binary search. There are $N(2 N-1)$ great circles in the arrangement $\mathcal{A}$ since every two points of $P$ lie on the unique great circle. The great circles of $\mathcal{A}$ intersect $\gamma$ in at most $N(2 N-1)$ points. These points partition $\gamma$ into the $\operatorname{arcs} A_{1}, A_{2}, \ldots, A_{M}$ where $M \leq N(2 N-1)+1$. One can apply the binary search on the arcs. Let $A_{i}, i=\lfloor M / 2\rfloor$, be the median arc and let $p$ be a point in $A_{i}$. Compute $\omega(p)$ and compare it with $\omega\left(\gamma_{0}\right)$ and $\omega\left(\gamma_{1}\right)$. One of the arcs $\gamma_{0} p$ or $p \gamma_{1}$ (or both) satisfies the property that its endpoints have different winding numbers. We can proceed with this arc. By Lemma 5 the algorithm finds an equitable 2 -fan.

We actually apply the binary search for two sub-arrangements of $\mathcal{A}$. Let $\mathcal{A}_{+}$, resp. $\mathcal{A}_{-}$, be the arrangement of the great circles passing through pairs of points $P \cap S_{+}$, resp. $P \cap S_{-}$, where $S_{+}=\{(x, y, z) \in S \mid z>0\}$ is the upper hemisphere of $S$ and $S_{-}=\{(x, y, z) \in S \mid z<0\}$ is the lower hemisphere of $S$. The binary search used in the algrithm is a combination of two searches in $\mathcal{A}_{+}$and $\mathcal{A}_{-}$, see details later. Our algorithm relies on the fact that the great circle $\gamma\left(p_{a}, p_{b}\right)$ generated by a point $p_{a}$ in $S_{+}$ and a point $p_{b}$ in $S_{-}$can be ignored since the path $\pi(p)$ does not change when $p$ crosses $\gamma \cap \gamma\left(p_{a}, p_{b}\right)$. Therefore we can restrict ourself to the arrangements $\mathcal{A}_{+}$and $\mathcal{A}_{-}$only. The question now is how to find the $i$ th arc on $\gamma$ generated by $\mathcal{A}_{+}$or $\mathcal{A}_{-}$. We show that this can be done using slope selection.

Slope selection. The points on the upper hemisphere $S_{+}$can be mapped to the plane $\Pi=\{(x, y, z) \mid z=1\}$ using the gnomonic projection $\eta: S_{+} \rightarrow \Pi$ defined as $\eta(x, y, z)=(x / z, y / z, 1)$. Let $p_{a}=\left(x_{a}, y_{a}, z_{a}\right)$ and $p_{b}=\left(x_{b}, y_{b}, z_{b}\right)$ be two points on the upper hemisphere and let $\gamma\left(p_{a}, p_{b}\right)$ denote the great circle containing $p_{a}$ and $p_{b}$. The great circle $\gamma\left(p_{a}, p_{b}\right)$ is mapped to the line $l\left(p_{a}, p_{b}\right)$ passing through the points $\eta\left(p_{a}\right)$ and $\eta\left(p_{b}\right)$. We show that the sorted order of the lines $l\left(p_{a}, p_{b}\right)$ by slope is in the one-to-one correspondence with the order of the points $\gamma \cap \gamma\left(p_{a}, p_{b}\right)$ on the half-circle $\gamma$. The slope of the line $l\left(p_{a}, p_{b}\right)$ is

$$
s=\frac{y_{b} / z_{b}-y_{a} / z_{a}}{x_{b} / z_{b}-x_{a} / z_{a}}
$$


Let $p_{\gamma}(x, y, 0)$ be the intersection point of the arc $\gamma$ and the great circle $\gamma\left(p_{a}, p_{b}\right)$. Since the points $p_{\gamma}, 0, p_{a}$ and $p_{b}$ are coplanar we have

$$
\left|\begin{array}{llll}
0 & 0 & 0 & 1 \\
x & y & 0 & 1 \\
x_{a} & y_{a} & z_{a} & 1 \\
x_{b} & y_{b} & z_{b} & 1
\end{array}\right|=0
$$

Then $x\left(y_{a} z_{b}-y_{b} z_{a}\right)=y\left(x_{a} z_{b}-x_{b} z_{a}\right)$ and the slope is $s=y / x$. Note that $y / x$ is $\tan \psi$ where $\psi$ is the angle of $0 p_{\gamma}$. The one-to-one correspondence between the slopes of the lines $l\left(p_{a}, p_{b}\right), p_{a} \in S_{+}, p_{b} \in S_{-}$, and the points $\gamma \cap \gamma\left(p_{a}, p_{b}\right)$ follows from the fact that $\psi \in(\pi / 2,3 \pi / 2)$ and $\tan ()$ is the monotone function in this interval.

Let $A_{1}^{+}, A_{2}^{+}, \ldots, A_{m^{+}}^{+}$be the arcs on $\gamma$ into which $\gamma$ is partitioned by the great circles of $\mathcal{A}_{+}$. Similarly, let $A_{1}^{-}, A_{2}^{-}, \ldots, A_{m^{-}}^{-}$be the arcs generated by $\mathcal{A}_{-}$. We assume that the $\operatorname{arcs} A_{i}^{+}$(and the arcs $A_{j}^{-}$) are in counterclockwise order. Using slope selection we can find the $i$ th $\operatorname{arc} A_{i}^{+}$. Slope selection can be done in $O(n \log n)$ time [8], [9] (a randomized algorithm can be found in [11] and [18]).

Binary search in two arrangements. In the binary search step we have the sequence of $\operatorname{arcs} A_{i_{1}}^{+}, A_{i_{1}+1}^{+}, \ldots, A_{i_{2}}^{+}$and the sequence of $\operatorname{arcs} A_{j_{1}}^{-}, A_{j_{1}+1}^{-}, \ldots, A_{j_{2}}^{-}$. At the beginning the indices are $i_{1}=j_{1}=1, i_{2}^{+}=m^{+}$and $j_{2}^{-}=m^{-}$. Using the slope selection we find two $\operatorname{arcs} A_{i}^{+}=\left(p_{i}^{+}, p_{i+1}^{+}\right)$and $A_{j}^{-}=\left(p_{j}^{-}, p_{j+1}^{-}\right)$where $i=\left\lfloor\left(i_{1}+i_{2}\right) / 2\right\rfloor$ and $j=\left\lfloor\left(j_{1}+j_{2}\right) / 2\right\rfloor$. Notice that the arc $A_{i}^{+}$may contain many arcs generated by $\mathcal{A}$. Therefore the points of $A_{i}^{+}$may have different winding numbers.

Suppose that $A_{i}^{+}$and $A_{j}^{-}$are disjoint. If the sequence of arcs of $\mathcal{A}_{+}$is narrowed to just the arc $A_{i}^{+}$, i.e. $i_{1}=i_{2}$, then we make the decision for $\mathcal{A}_{-}$. In the example depicted in Fig. 5(a), the indices of the arcs in $\mathcal{A}_{-}$should be less than $j$. If the sequence of arcs of $\mathcal{A}_{+}$has more than one segment then we pick a point $p$ in the arc $A_{i}^{+}$infinitesimally close to $p_{i+1}^{+}$and test it. If $\omega(p)=\omega\left(\gamma_{0}\right)$ then we prune the $\operatorname{arcs}$ in $\mathcal{A}_{+}$with indices less than $i$. Otherwise we prune the $\operatorname{arcs}$ in $\mathcal{A}_{+}$with indices greater than $i$ and the $\operatorname{arcs}$ in $\mathcal{A}_{-}$ with indices greater than $j$.

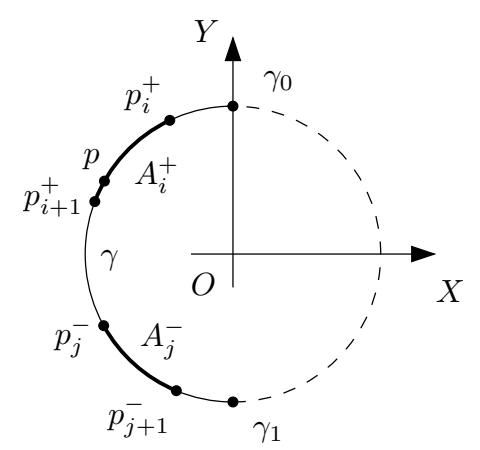

(a)

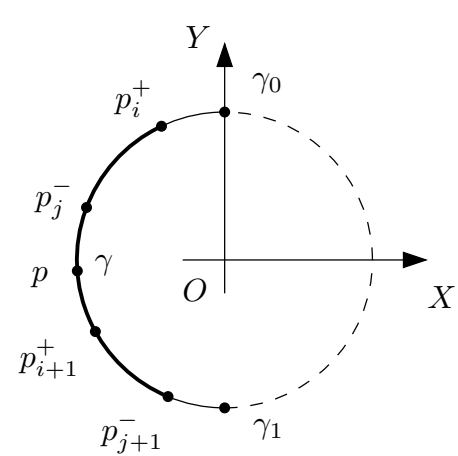

(b)

Fig. 5. The binary search step. 
If the $\operatorname{arcs} A_{i}^{+}$and $A_{j}^{-}$intersect then we select any point $p$ from their intersection, see Fig. 5(b). Note that all the points of $A_{i}^{+} \cap A_{j}^{-}$have the same winding number since the path $\pi(p)$ does not change when $p$ crosses $\gamma \cap \gamma\left(p_{a}, p_{b}\right)$ for any $p_{a} \in S_{+}, p_{b} \in S_{-}$. The binary search test for $p$ prunes either the arcs of $\mathcal{A}_{+}$or $\mathcal{A}_{-}$(or both).

The total number of the binary search tests is $O(\log N)$ since at least one quarter of the arcs is pruned at every binary search step. The theorem follows.

The problem of finding an equitable 2-fan on the sphere is more general than the one in the plane since we can map the plane $\Pi=\{(x, y, z) \mid z=1\}$ to the sphere $S$ using the inverse gnomonic projection $\eta^{-1}$ which maps a plane point $(x, y, 1)$ to the sphere point $(x z, y z, z)$, where $z=1 / \sqrt{x^{2}+y^{2}+1}$. As an immediate consequence we obtain the following result.

Theorem 8. Given a set of $2 N$ points in the plane colored in red/blue/green such that (i) the points are in general position, and (ii) the number of points of each color is even, an equitable 2-fan with center on a given line can be found in $O\left(N \log ^{2} N\right)$ time using $O(N)$ space.

\section{Conclusion}

We proved that any three mass measures on the 2-sphere admit an equitable partition by a 2 -fan whose center lies on a given great circle. Based on our proof we showed that an equitable 2-fan can be computed in $O\left(N \log ^{2} N\right)$ time for discrete masses. The complexity of the problem is an interesting open question: one can either improve the running time (a new proof may be needed for this) or/and find a non-trivial lower bound.

\section{References}

1. J. Akiyama, A. Kaneko, M. Kano, G. Nakamura, E. Rivera-Campo, S. Tokunaga, and J. Urrutia. Radial perfect partitions of convex sets in the plane. In Proc. Japan Conf. Discrete Comput. Geom. '98, pp. 1-13. Volume 1763 of Lecture Notes Computer Science. Springer-Verlag, Berlin, 2000.

2. I. Bárány. Geometric and combinatorial applications of Borsuk's theorem. In J. Pach, editor, New Trends in Discrete and Computational Geometry, pp. 235-249. Volume 10 of Algorithms and Combinatorics. Springer-Verlag, Berlin, 1993.

3. I. Bárány and J. Matoušek. Simultaneous partitions of measures by $k$-fans. Discrete Comput. Geom., 25(3):317-334, 2001.

4. I. Bárány and J. Matoušek. Equipartition of two measures by a 4-fan. Discrete Comput. Geom., 27(3):293$301,2002$.

5. M. W. Bern and D. Eppstein. Multivariate regression depth. Discrete Comput. Geom., 28(1):1-17, 2002.

6. S. Bespamyatnikh, D. Kirkpatrick, and J. Snoeyink. Generalizing ham sandwich cuts to equitable subdivisions. Discrete Comput. Geom., 24(4):605-622, 2000. http://springerlink.metapress.com/ openurl.asp?genre $=$ article $\&$ issn $=0179-5376 \&$ volume $=24 \&$ issue $=4 \&$ spage $=605$.

7. P. Bose, E. D. Demaine, F. Hurtado, J. Iacono, S. Langerman, and P. Morin. Geodesic ham-sandwich cuts. In Proc. 20th Annu. ACM Sympos. Comput. Geom., pp. 1-9, 2004. http://www-ma2.upc.es/ hurtado/ham.pdf.

8. H. Brönnimann and B. Chazelle. Optimal slope selection via cuttings. Comput. Geom. Theory Appl., 10(1):23-29, 1998. 
9. R. Cole, J. Salowe, W. Steiger, and E. Szemerédi. An optimal-time algorithm for slope selection. SIAM J. Comput., 18(4):792-810, 1989.

10. M. Díaz and J. O'Rourke. Ham-sandwich sectioning of polygons. In Proc. 2nd Canad. Conf. Comput. Geom., pp. 282-286, 1990.

11. M. B. Dillencourt, D. M. Mount, and N. S. Netanyahu. A randomized algorithm for slope selection. Internat. J. Comput. Geom. Appl., 2:1-27, 1992.

12. D. P. Dobkin and H. Edelsbrunner. Ham-sandwich theorems applied to intersection problems. In Proc. 10th Internat. Workshop Graph-Theoret. Concepts Comput. Sci., pp. 88-99, 1984.

13. H. Edelsbrunner and R. Waupotitsch. Computing a ham-sandwich cut in two dimensions. J. Symbolic Comput., 2:171-178, 1986.

14. H. Ito, H. Uehara, and M. Yokoyama. Two-dimension ham sandwich theorem for partitioning into three convex pieces. In Proc. Japan Conf. Discrete Comput. Geom.'98, pp. 129-157. Volume 1763 of Lecture Notes Computer Science. Springer-Verlag, Berlin, 2000.

15. A. Kaneko and M. Kano. Balanced partitions of two sets of points in the plane. Comput. Geom. Theory Appl., 13:253-261, 1999.

16. M. Kano. Personal communication.

17. C.-Y. Lo, J. Matoušek, and W. L. Steiger. Algorithms for ham-sandwich cuts. Discrete Comput. Geom., 11:433-452, 1994.

18. J. Matoušek. Randomized optimal algorithm for slope selection. Inform. Process. Lett., 39:183-187, 1991.

19. J. Matoušek. Using the Borsuk-Ulam Theorem. Springer-Verlag, Heidelberg, 2003.

20. T. Sakai. Balanced convex partitions of measures in $\mathbb{R}^{2}$. Graphs Combin., 18(1):169-192, 2002.

21. W. Steiger. Algorithms for ham sandwich cuts. In Proc. 5th Canad. Conf. Comput. Geom., p. 48, 1993.

22. S. T. Vrećica and R. T. Živaljević. Conical equipartitions of mass distributions. Discrete Comput. Geom., 25(3):335-350, 2001.

23. R. T. Živaljević. Topological methods. In J. E. Goodman and J. O'Rourke, editors, Handbook of Discrete and Computational Geometry, chapter 11, pp. 209-224. CRC Press, Boca Raton, FL, 1997.

24. R. T. Živaljević and S. T. Vrećica. An extension of the ham sandwich theorem. Bull. London Math. Soc., 22:183-186, 1990.

Received June 5, 2004, and in revised form July 15, 2004. Online publication January 21, 2005. 